\title{
MOVING MESH METHODS FOR PROBLEMS WITH BLOW-UP*
}

\author{
CHRIS J. BUDD ${ }^{\dagger}$, WEIZHANG HUANG ${ }^{\ddagger}$, AND ROBERT D. RUSSELL ${ }^{\S}$
}

\begin{abstract}
In this paper we consider the numerical solution of PDEs with blow-up for which scaling invariance plays a natural role in describing the underlying solution structures. It is a challenging numerical problem to capture the qualitative behaviour in the blow-up region, and the use of nonuniform meshes is essential. We consider moving mesh methods for which the mesh is determined using so-called moving mesh partial differential equations (MMPDEs). Specifically, the underlying PDE and the MMPDE are solved for the blow-up solution and the computational mesh simultaneously. Motivated by the desire for the MMPDE to preserve the scaling invariance of the underlying problem, we study the effect of different choices of MMPDEs and monitor functions. It is shown that for suitable ones the MMPDE solution evolves towards a (moving) mesh which close to the blow-up point automatically places the mesh points in such a manner that the ignition kernel, which is well known to be a natural coordinate in describing the behaviour of blow-up, approaches a constant as $t \rightarrow T$ (the blow-up time). Several numerical examples are given to verify the theory for these MMPDE methods and to illustrate their efficacy.
\end{abstract}

Key words. blow-up solution, moving mesh, scaling invariance

AMS subject classifications. 35K55, 65M50, 35B40, 65M20

1. Introduction. Many mathematical idealizations of physical models have the property that they develop singularities in a finite time $T$. Examples are the blow-up of the solutions of models describing combustion in chemicals or chemotaxis in cellular aggregates and the formation of shocks in the inviscid Burgers' equation and the space-charge equations. Such a singularity often represents an important change in the properties of the model, such as the ignition of a heated gas mixture, and it is important that it should be accurately reproduced by a numerical computation.

When a singularity forms, changes occur on increasingly small length scales and, as the time $T$ is approached, on increasingly smaller timescales. If a numerical method with a fixed mesh is used to reproduce such behaviour then its accuracy will diminish significantly when the length scale of the singularity approaches the spacing between mesh points. In some cases this will lead to numerical solutions which differ qualitatively from the underlying analytic solution. Indeed, examples can be found where a computation on a fixed mesh misses the blow-up entirely, or where the numerical solution blows up over the whole region even though the analytic solution develops a singularity at a single point [AB94].

To compute such singular behaviour accurately, it is essential to use a numerical method which adapts the spatial mesh as the singularity develops. Ideally, the numerical method will reproduce the singularity sufficiently accurately as $t \rightarrow T$ to mimic the asymptotic behaviour of the solution. A feature of a wide class of PDEs (partial differential equations) which makes this feasible is that the spatial structure of the singularity evolves in a fairly simple manner, often independent of any local structure in the initial conditions. Provided that the adaptive method can reproduce this simple asymptotic behaviour, there is reason to hope that a numerical scheme can be designed to perform accurately for all times $t<T$.

One class of problems which have this feature is the semilinear parabolic equations describing the blow-up of the temperature of a reacting medium, such as a burning gas. The

${ }^{*}$ Received by the editors February 14, 1994; accepted for publication November 28, 1994. This work was supported in part by NSERC grant OGP-0008781, the Nuffield Foundation, and SERC grant GR/J56219.

${ }^{\dagger}$ School of Mathematics, University of Bristol, Bristol BS8 1TW, U.K. (Chris.Budd@ bristol.ac.uk).

$\ddagger$ Department of Mathematics and Statistics, Simon Fraser University, Burnaby, B.C. V5A 1S6, Canada. Current address: Department of Mathematics, 405 Snow Hall, University of Kansas, Lawrence, KS 66045 (whuang@math.ukans.edu).

$\S$ Department of Mathematics and Statistics, Simon Fraser University, Burnaby, B.C. V5A 1S6, Canada (rdr@cs.sfu.ca). 
simplest equations describing such blow-up have the form

$$
u_{t}=u_{x x}+f(u), u(0, t)=u(1, t)=0, u(0, x)=u_{0}(x),
$$

where $f(u)$ is any convex function of $u$ such that $f(u) \rightarrow \infty$ as $u \rightarrow \infty$. In this paper we shall consider the cases of $f(u)=u^{p}, f(u)=e^{u}$, and slightly more general problems. It is well known [FM85] that if $u_{0}(x)$ is "sufficiently large" and has a single nondegenerate maximum, then there is a blow-up time $T<\infty$ and a unique blow-up point $x^{*}$ such that

$$
u\left(x^{*}, t\right) \rightarrow \infty \text { as } t \rightarrow T
$$

and

$$
u(x, t) \rightarrow u(x, T)<\infty \text { if } x \neq x^{*} .
$$

(If $t>T$ the solution becomes infinite everywhere.) Close to $x^{*}$, the solution $u(x, t)$ develops an isolated peak which becomes narrower, tending to zero width, as $t \rightarrow T$. A derivation and general study of these systems is given in [BE89].

The computation of the solutions of (1.1) is important for several reasons. First, although very simple, the formation of the singularities in this problem is typical of that of a wide class of PDEs modelling many differing physical phenomena. Second, a great deal is known about the analytic structure of the solutions of (1.1) for $t$ close to $T$ and $x$ close to $x^{*}$, and thus they make excellent problems for testing the performance of and verifying the analysis for the numerical methods used in their solution. Third, having numerical methods which are faithful to the underlying asymptotics of the PDEs raises the possibility of solving very difficult problems for which the analytic structure is unknown, and then using the resulting numerical solutions to lend insight into this structure. Of course this can in turn motivate derivation of further theoretical results.

Existing adaptive numerical methods for solving (1.1) are described in [Cho81], [LPSS86], [BK88], [Ber89], and [BDS93]. These are either based upon closely exploiting the known analytic structure of the singularity or on an adaptive procedure which requires an increasingly larger number of mesh points to model the developing singularity as $t \rightarrow T$. In contrast, we shall describe here an elegant set of methods for solving (1.1) which use the dynamic gridding algorithms described in [HRR94b]. These MMPDE (moving mesh PDE) methods are based upon equidistributing a monitor function, say $M\left(u, u_{x}, u_{x x}\right)$, which relies on no a priori knowledge of the solution to the PDE $u$, although an analysis of scaling properties of the PDE does lead to certain optimal choices of $M$. We shall show that these methods have the significant property that they reproduce the dynamical nature of the development of the singularity. In particular, there is a natural rescaling of the spatial coordinate close to the singularity which is automatically captured by the moving mesh method.

The asymptotic scaling of the singularity was first observed formally by [Dol85]. A full proof is given in [BB92]. They showed that for $x$ close to $x^{*}$ and $t$ close to $T$ there was a natural spatial coordinate, the so-called ignition kernel $\mu$, where

$$
\mu(x, t)=\left(x-x^{*}\right)[(T-t)|\log (T-t)|]^{-\frac{1}{2}} .
$$

In [BK88] the original PDE is recast in terms of the closely related "similarity" variable

$$
\zeta=\mu \log ^{\frac{1}{2}}(T-t)
$$

which is derived from a scaling invariance of the original PDE, and the resulting scaled PDE is then solved by using a static regridding algorithm. In contrast, we show that the MMPDE 
solution evolves towards a (moving) mesh which close to the singularity automatically places the mesh points $x_{i}(t)$ in such a manner that $\mu\left(x_{i}, t\right)$ approaches a constant as $t \rightarrow T$. In this way, the MMPDE methods naturally inherit the correct spatial structure of the singularity.

The paper is organized as follows. In $\S 2$ we introduce the blow-up problems and describe the asymptotic form of solutions close to blow-up. In $\S 3$ we introduce the MMPDE method for determining the mesh coordinate transformation used to solve these problems. Motivated by the desire to preserve scaling invariance, we consider the effect of different choices of the MMPDE and monitor function $M$. In $\S 4$ we show that in fact the "approximate" similarity solution behaviour of (1.1) is preserved by suitable choices of $M$, using techniques for the discrete analysis which mimic the previous analyses for the continuous case. In $\S 5$ we give numerical examples to verify the theory for these MMPDE methods and illustrate their efficacy. Finally, in $\S 6$ we state some conclusions and briefly discuss a more general framework under current investigation.

2. Structure of blow-up solutions for PDEs. We now consider the form of the solutions of the semilinear parabolic PDE

$$
\begin{aligned}
& u_{t}=u_{x x}+u^{p}, \quad p>1 \\
& u(0, t)=u(1, t)=0 \\
& u(x, 0)=u_{0}(x)>0
\end{aligned}
$$

As well as looking at the asymptotic form of the solutions close to blow-up we also consider some of the underlying principles which lead to these solutions. These principles can then be used as a guide to the numerical method and also to study other related equations.

It has been shown by several authors, e.g., [FM85], that if $u_{0}(x)$ is sufficiently large and positive and has a single nondegenerate maximum, then (1.2) and (1.3) hold. The point $x^{*}$ and the time $T$ depend subtlely upon $u_{0}(x)$ but, remarkably, the solution $u(x, t)$ itself is almost independent of $u_{0}$ provided that $x$ and $t$ are close to $x^{*}$ and $T$, respectively. The blow-up profile takes the form of an isolated spike of increasingly narrow width and has been studied by [Dol85] and [BK88]. The behaviour of this spike may be described as follows.

THEOREM 2.1. Let $\beta=\frac{1}{p-1}$ and let $\mu(x, t)$ be defined by

$$
\mu=\left(x-x^{*}\right)[(T-t)(\alpha-\log (T-t))]^{-\frac{1}{2}}
$$

where $\alpha$ is a constant which depends on $u_{0}(x)$.

(i) If $x(t)$ is taken to keep $\mu(x, t)$ constant, then the solution $u(x, t)$ to $(2.1)$ satisfies

$$
(T-t)^{\beta} u(x, t) \rightarrow \beta^{\beta}\left[1+\frac{\mu^{2}}{4 p \beta}\right]^{-\beta} \text { as } t \rightarrow T .
$$

(ii) If $\left|x-x^{*}\right|$ is small but fixed and independent of $t$, then

$$
u(x, t) \rightarrow u(x, T)=\left[4 p \beta^{2} \frac{|\alpha-2 \log | x-x^{*}||}{\left|x-x^{*}\right|^{2}}\left(1+O\left(\left|x-x^{*}\right|\right)^{2}\right)\right]^{\beta}
$$

as $t \rightarrow T$.

We observe that the expressions (2.3) and (2.4) coincide if we set $\mu$ to be large. The expression (2.3) describes the evolution of the blow-up peak in terms of the "ignition kernel" $\mu$. This variable was first identified by [Dol85], and is a natural variable to describe the spatial structure of blow-up. A remarkable feature of the numerical methods we shall describe is that close to $x^{*}$ the moving mesh is placed precisely at those points for which $\mu$ is constant. 
The starting point for deriving these expressions is a natural scaling invariance of the solutions of (2.1) (in the absence of boundary conditions). In particular, the equation is invariant under the scaling

$$
\left\{\begin{array}{l}
(T-t) \rightarrow \lambda(T-t) \\
u \rightarrow \frac{1}{\lambda^{\beta}} u \\
x \rightarrow \lambda^{\frac{1}{2}} x
\end{array}\right.
$$

for any positive $\lambda$. A similarity solution of (2.1) is any solution which is invariant under this scaling. Many interesting PDEs, including problems leading to blow-up (see below), have a scaling invariance similar to (2.5).

Motivated by (2.5) we recast (2.1) in terms of similarity variables $w(s, y), y$, and $s$ defined by

$$
\left\{\begin{array}{l}
s=-\log (T-t) \\
w(s, y)=(T-t)^{\beta} u(x, t), \\
y=\left(x-x^{*}\right)(T-t)^{-1 / 2}
\end{array}\right.
$$

to give the partial differential equation

$$
w_{s}=w_{y y}-\frac{1}{2} y w_{y}+w^{p}-\beta w
$$

supplemented with

$$
w_{y}(s, 0)=0 \text { and } w(s, y) \rightarrow 0 \text { as }|y| \rightarrow \infty .
$$

The latter condition is necessary to match the boundary conditions satisfied by the solutions of the unscaled problem.

A similarity solution of the original PDE is a steady state (i.e., $s$ independent) solution of (2.7) which also satisfies (2.8). These solutions were originally proposed as solutions of (2.1), but in fact, the only bounded, nonzero steady state solution of (2.7) is

$$
w(s, y)=\beta^{\beta},
$$

which fails to satisfy (2.8). However, if we consider a set of points $x(t)$ such that $y(x, t)$ is fixed, then by using energy arguments Giga and Kohn [GK85] show that

$$
(T-t)^{\beta} u(x, t) \rightarrow \beta^{\beta},
$$

so that the function (2.9) is an attractor for solutions of (2.7) over compact sets in $y$.

To calculate a solution of (2.7) which corresponds to a solution of (2.1) we consider instead a perturbation of the similarity solution by setting

$$
w(s, y)=f\left(\frac{y}{g(s)}\right) \equiv f(z)
$$

where $f$ and $g$ satisfy the conditions

$$
\left\{\begin{array}{l}
f(z) \rightarrow 0 \text { as }|z| \rightarrow \infty \\
g(s) \rightarrow \infty \text { as } s \rightarrow \infty \\
\frac{g^{\prime}}{g} \rightarrow 0 \text { as } s \rightarrow \infty
\end{array}\right.
$$


The derivation of these conditions is given in [BK88]. Substituting into (2.7) gives

$$
-\frac{g^{\prime}}{g} z f_{z}-g^{-2} f_{z z}+\frac{1}{2} z f_{z}+\beta f-f^{p}=0
$$

which, for large $s$, reduces to the first-order equation

$$
\frac{1}{2} z f_{z}+\beta f-f^{p}=0
$$

with solution

$$
f(z)=\left[\frac{1}{\beta}+c z^{2}\right]^{-\beta},
$$

where $c$ is a constant which without loss of generality may be set to 1 . The reduction of problem (2.1) from a second-order equation (2.7) to a first-order Hamilton-Jacobi one (2.14) is a crucial feature of blow-up problems, see [GV93]. The function $g$, giving the spreading rate, can be derived formally by making an expansion of the function $w$ in powers of $\frac{1}{s}$ and matching terms. This implies that $g^{-2}, \frac{g^{\prime}}{g}$, and $s^{-1}$ should all be of the same order for $s>>1$. The details of this derivation are given in [BK88] and [Dol85] and give

$$
g(s)=\left[4 \beta^{2} p(s+\alpha)\right]^{\frac{1}{2}}
$$

where $\alpha$ is a constant which depends (weakly) upon the initial conditions. Combining (2.15) with (2.16) and taking $s$ large gives (2.3).

The derivation given here is formal and is closely related to an analysis of the behaviour of the numerical scheme described in $\S 4$. A more precise derivation of the result (2.3) describing the shape of the blow-up peak follows from a centre-manifold reduction of the solutions of (2.7) and is given in [BB92], [FK92], and [HV93]. These papers also give a rigorous derivation of (2.4), although this equation follows formally from taking the large $\mu$ limit of (2.3) and matching to a steady state solution.

A useful conclusion from (2.3) is the natural relationship between the various scalings involved in the solution of the blow-up problem. In particular, if we consider $\tau=(T-t)$ to be the local "timescale" for blow-up, then the corresponding scale for $u$ is

$$
U=\frac{1}{\tau^{\beta}}
$$

and the length scale for $x$ is

$$
X=[\tau(\alpha-\log \tau)]^{1 / 2}
$$

Furthermore, the scale for $u_{x}$ is approximately given by

$$
u_{x} \approx \frac{U}{X}=\left[\tau^{1+2 \beta}(\alpha-\log \tau)\right]^{-1 / 2}
$$

These scales are useful in deciding the choice of an appropriate numerical method. For example, (2.18) gives an indication of the correct mesh spacing close to the blow-up point.

The scaling invariance (2.5) plays a crucial role in the analysis of (2.1). Although the solution of the PDE is not self-similar, it is close to being self-similar and moreover converges to the self-similar solution $w=\beta^{\beta}$ on any compact interval in the similarity variable $y$. 
Such scaling invariance and associated self-similar and approximately self-similar solution behaviour is found in many other equations describing blow-up and for completeness we list some of these here. Further examples of semilinear and quasi-linear PDEs related to (2.1) include the Kassoy problem [Kas77], [Gel63]

$$
u_{t}=u_{x x}+e^{u},
$$

and porous-medium reaction-diffusion equations

$$
u_{t}=\left(\left|u_{x}\right|^{\sigma} u_{x}\right)_{x}+e^{u}
$$

and

$$
u_{t}=\left(u^{\alpha} u_{x}\right)_{x}+u^{p}
$$

which are analyzed in [BDG93] and in [GP91], respectively. Furthermore, the nonlinear Schrödinger equation [LPSS86]

$$
i \psi_{t}+\Delta \psi+|\psi|^{2 \sigma} \psi=0
$$

is an example of a hyperbolic PDE for which blow-up occurs in $R^{N}$ if $N \geq 2$, and this has been used to model focusing in lasers.

The similarity variables for (2.20) and (2.21) are

$$
\left\{\begin{array}{l}
w(s, y)=\log (T-t)+u(x, t) \\
y=x(T-t)^{-\frac{1}{\sigma+2}}
\end{array}\right.
$$

and with this change of variables (2.20) and (2.21) reduce to PDEs similar to (2.7), which can be analyzed in an analogous way. Remarkably, the corresponding solution is approximately self-similar if $\sigma=0$ and exactly self-similar if $\sigma>0$. Similar behaviour to that in (2.1) is observed for (2.22) with $\alpha>0$, where

$$
y=x(T-t)^{-\frac{1}{2}[1+\alpha /(p-1)]} .
$$

The behaviour of the nonlinear Schrödinger equation is less well understood, but it is invariant under various scalings. In particular, it has a natural set of similarity variables given by

$$
\left\{\begin{array}{l}
y=\frac{r}{L(t)}, \\
\tau=\int_{0}^{t} \frac{d s}{L}, \\
u(y, \tau)=L^{1 / \sigma} \psi,
\end{array}\right.
$$

where $r=|x|$ and $L(t)$ can be chosen in various ways. These follow from the scaling invariance of solutions given by

$$
\psi(x, t) \rightarrow \lambda^{-1 / \sigma} \psi\left(\frac{x}{\lambda}, \frac{t}{\lambda^{2}}\right) .
$$

In [LPSS86] extensive use is made of this rescaling in calculating the solution of (2.23) numerically. Self-similar solutions arise when $L(t)=(T-t)^{1 / 2}$, as in the previous problems, and numerical evidence for the existence of such solutions has been obtained.

It is clear from this brief discussion that the scaling invariance of (2.1) and (2.20) - (2.23) plays a key role in determining the dynamical solution behaviour. This strongly implies that numerical methods which respect this invariance should be more effective in reproducing the dynamics than those which do not. Such methods must necessarily employ moving meshes to allow for rescalings in both space and the solution. We now consider these. 
3. Moving mesh PDEs with scaling invariance. The class of methods which we propose for solving (1.1) are the moving mesh PDE methods described in [HRR94a] and [HRR94b]. For these the function $u(x, t)$ is discretized to give the solution values $u_{i}(t)$ defined on a moving mesh $x_{i}(t), i=0, \ldots, N$. The boundary conditions in (1.1) give $u_{0}=u_{N}=0$ and $x_{0}=0, x_{N}=1$. The mesh $x_{i}(t)$ is defined in terms of a differentiable mesh transformation $x(\xi, t):[0,1] \rightarrow[0,1]$, where $x$ is the physical coordinate and $\xi$ is the computational coordinate such that

$$
x_{i}(t)=x\left(\frac{i}{N}, t\right)
$$

In the continuous formulation, the constraint

$$
\frac{\partial x}{\partial \xi}>0
$$

assures that the mesh transformation is well defined for fixed $t$, and the discrete analogue is that mesh crossing does not occur. For the MMPDE approach, a new partial differential equation for $x(\xi, t)$, called the moving mesh PDE, is solved simultaneously with the original PDE for $u(x, t)$. The underlying strategy for determining $x(\xi, t)$ is to require equidistribution of a positive monitor function, say $M\left(u, u_{x}, u_{x x}\right)$, so that

$$
\int_{0}^{x} M d y=\xi \int_{0}^{1} M d y .
$$

Equivalently, differentiating this identity gives

$$
\frac{\partial}{\partial \xi}\left(M \frac{\partial x}{\partial \xi}\right)=0, x(0, t)=0, x(1, t)=1 .
$$

A mesh (or a coordinate transformation) is said to be equidistributed when (3.4) holds. It is convenient in practice not to enforce exact equidistribution upon a mesh but to instead solve an MMPDE for which it tends toward an equidistributed state. This has the advantages that a simple initial mesh (such as a uniform one) can be used, the process produces stable meshes with less risk of mesh crossing than if (3.3) were enforced, and combined with a smoothing approach it reduces the problem (associated, for example, with the schemes proposed in [LPSS86]) of placing so many points close to the developing singularity that resolution is lost elsewhere. Of the various MMPDEs proposed in [HRR94a], we consider the two labelled MMPDE4 and MMPDE6. These are, respectively,

(MMPDE4)

$$
\tau \frac{\partial}{\partial \xi}\left(M \frac{\partial \dot{x}}{\partial \xi}\right)=-\frac{\partial}{\partial \xi}\left(M \frac{\partial x}{\partial \xi}\right)
$$

and

(MMPDE6)

$$
\tau \frac{\partial^{2} \dot{x}}{\partial \xi^{2}}=-\frac{\partial}{\partial \xi}\left(M \frac{\partial x}{\partial \xi}\right)
$$

Here, $\dot{x}$ denotes $\left.\frac{\partial x}{\partial t}\right|_{\xi}$ fixed and $\tau$ is a small parameter which acts to relax the mesh to the equidistributed state. Note that MMPDE6 is the second derivative with respect to $\xi$ of the integro-differential equation

$$
\tau \dot{x}=-\left(\int_{0}^{x} M d x-\xi \int_{0}^{1} M d x\right) .
$$


It is worth remarking that many previous moving mesh equations can be regarded as variants of or discrete approximations of these MMPDEs. (See, e.g., [HL89] for MMPDE6; see also [HRR94a] and [HRR94b].)

If we apply moving mesh PDE methods to solve a PDE with an underlying scaling invariance, then as the underlying PDE and the MMPDE are solved simultaneously to determine both the solution $u$ and the mesh $x$, it is desirable that the scaling invariance of the underlying PDE be preserved. For (2.1) this requires that MMPDE4 or MMPDE6 be invariant under the scaling (2.5). The parameter $\tau$ and the monitor function $M$ can indeed be suitably chosen to meet this requirement. Assuming that the solution $u(x, t)$ to $(2.1)$ is positive for $x \in(0,1)$ and $t \geq 0$, MMPDE6 can be made invariant under the scaling (2.5) if $\tau$ is taken as a dimensionless constant and

$$
M(u)=u^{p-1} .
$$

However, regardless of the choice of $M, \tau$ cannot be constant if MMPDE4 is to be invariant under (2.5) (although we can obtain an invariance if $\tau$ is chosen adaptively).

This difference between MMPDE6 and MMPDE4 becomes important when we consider the timescales under which the mesh adapts to follow the structure of the solution. An inspection of MMPDE4 and MMPDE6 shows that each has a natural timescale $T_{\text {mesh }}$ for adapting the mesh towards an equidistributed mesh. For MMPDE4 $T_{\text {mesh }}=O(\tau)$ and for MMPDE6 $T_{\text {mesh }}=O\left(\frac{\tau}{M}\right)$. If $T_{\text {mesh }}$ is significantly greater than the natural timescale for the evolution of the solution structure, then the mesh cannot adapt rapidly enough to follow the solution structure and is to all intents and purposes fixed. As we showed in $\S 2$, the natural timescale for the evolution of the blow-up peak is $O(T-t)$. Hence, if

$$
T-t<<\tau,
$$

then MMPDE4 will not be able to evolve the mesh rapidly enough to follow the evolution of the peak. In contrast, if we look at MMPDE6 with $M$ as in (3.6), then $\frac{\tau}{M}=\frac{\tau}{u^{p-1}}=\tau(T-t)$. Hence the timescale for the evolution of the mesh is always a factor $\tau$ smaller than the natural timescale of the underlying problem, and MMPDE6 will continue to evolve the mesh for $t$ close to $T$. This is a direct consequence of the scaling invariance of MMPDE6 under this choice of monitor function. Other choices of monitor function (for example, arclength) do not share this property, and we will study this in more detail in $\S 4$.

For the numerical computation, PDE (2.1) is transformed in terms of the computational coordinate $\xi$ and discretized by central finite differences on a uniform mesh in the computational domain. That is, (2.1) is first transformed into the quasi-Lagrangian form

$$
\left\{\begin{array}{l}
\dot{u}-\frac{u_{\xi}}{x_{\xi}} \dot{x}=\frac{1}{x_{\xi}}\left(\frac{u_{\xi}}{x_{\xi}}\right)_{\xi}+u^{p}, \\
u(0, t)=u(1, t)=0,
\end{array}\right.
$$

and discretization gives the equation

$$
\dot{u}_{i}-\frac{u_{i+1}-u_{i-1}}{x_{i+1}-x_{i-1}} \dot{x}_{i}=\frac{2}{x_{i+1}-x_{i-1}}\left(\frac{u_{i+1}-u_{i}}{x_{i+1}-x_{i}}-\frac{u_{i}-u_{i-1}}{x_{i}-x_{i-1}}\right)+u_{i}^{p},
$$

$i=1, \ldots, N-1$ and $u_{0}=u_{N}=0$. Similar finite difference equations can be obtained for discretizations of MMPDEs 4 and 6.

It is well known that for moving mesh methods, some sort of smoothing of the mesh is often necessary in order to obtain nonoscillatory, reasonably accurate solutions (e.g., see [DD87], [FVZ90], and [HRR94b]). In [DD87], Dorfi and Drury use a technique which smooths the 
node concentration defined by $\frac{1}{x_{i+1}-x_{i}}$. In [VBFZ89], Verwer et al. prove that smoothing the node concentration is basically equivalent to smoothing the monitor function over all points. To maintain the local structure of the underlying difference equations, we use the technique employed in [HRR94b]. Specifically, the values of the smoothed monitor function $\tilde{M}$ at nodes are defined by

$$
\tilde{M}_{i}=\frac{\sum_{k=i-i_{p}}^{i+i_{p}} M_{k}\left(\frac{\gamma}{\gamma+1}\right)^{|k-i|}}{\sum_{k=i-i_{p}}^{i+i_{p}}\left(\frac{\gamma}{\gamma+1}\right)^{|k-i|}}
$$

where $M_{i} \equiv M\left(\xi_{i}, t\right), i_{p}$ is a nonnegative integer, and $\gamma$ is a positive constant. The summations in (3.10) are understood to contain only elements with indices in the range between 0 and $N$. Notice that the replacement of $M_{i}$ by $\tilde{M}_{i}$ is basically equivalent to using a smoother monitor function, and $i_{p}=0$ corresponds to the nonsmoothing case. Values of the parameters $\gamma$ and $i_{p}$ need to be selected for these moving mesh PDE methods. In our experience (also see [HRR94b]), the choice of $\gamma$ is fairly insensitive and can generally be fixed. In this paper, we use $\gamma=2$. The value for $i_{p}$ usually is taken as $0,1,2,3$, or 4 .

The final forms for the discrete moving mesh equations for MMPDE4 and MMPDE6 are

$$
\begin{aligned}
& \tau\left(\tilde{M}_{i+\frac{1}{2}}\left(\dot{x}_{i+1}-\dot{x}_{i}\right)-\tilde{M}_{i-\frac{1}{2}}\left(\dot{x}_{i}-\dot{x}_{i-1}\right)\right) \\
& =-\left(\tilde{M}_{i+\frac{1}{2}}\left(x_{i+1}-x_{i}\right)-\tilde{M}_{i-\frac{1}{2}}\left(x_{i}-x_{i-1}\right)\right),
\end{aligned}
$$

and

$$
\tau\left(\dot{x}_{i+1}-2 \dot{x}_{i}+\dot{x}_{i-1}\right)=-\left(\tilde{M}_{i+\frac{1}{2}}\left(x_{i+1}-x_{i}\right)-\tilde{M}_{i-\frac{1}{2}}\left(x_{i}-x_{i-1}\right)\right)
$$

$i=1, \ldots, N-1$ supplemented with $x_{0}=0, x_{N}=1$, where $\tilde{M}_{i+\frac{1}{2}}:=\left(\tilde{M}_{i}+\tilde{M}_{i+1}\right) / 2$. We note that the smoothing process maintains the dimension of the monitor function, which will become important when we consider rescalings of the equations.

4. Analysis of the solutions of the moving mesh equations. We now analyze the solution behaviour for the discretization of problem (2.1), using the discrete version of MMPDE6 together with the monitor function given by (3.6). The analysis will be in two parts. First, we shall solve MMPDE6 exactly assuming that $u(x, t)$ is as described in $\S 2$. This will, in effect, determine the "optimum" mesh for such a problem. Second, we shall analyze the solutions of the coupled finite difference equations (3.9) and (3.12), assuming exact time integration, and compare the solutions with those obtained in the continuous case.

4.1. Analysis of the moving mesh equations. Integrating MMPDE6 with respect to $\xi$, we know that the mesh transformation $x(\xi, t)$ satisfies the equation

$$
-\tau \dot{x}_{\xi}=M x_{\xi}+\theta(t)
$$

where $\theta(t)$ is an integral constant which will be determined by the boundary conditions for $x(\xi, t)$. Indeed, integrating (4.1) with respect to $\xi$ we have

$$
-\left.\tau \dot{x}(\xi, t)\right|_{\xi=0} ^{1}=\int_{0}^{1} M x_{\xi} d \xi+\theta(t) .
$$


Then, from the boundary conditions

$$
x(0, t)=x(1, t)-1=0,
$$

(4.2) implies that

$$
\theta(t)=-\int_{0}^{1} M d x
$$

Because the function $u(x, t)$ has a sharp peak, the integral above is asymptotically dominated by the contribution from the peak. It follows from (3.6) and (2.3) that within the blow-up region $M$ has the asymptotic form

$$
M=\frac{\beta}{(T-t)\left[1+\frac{\mu^{2}}{4 p \beta}\right]},
$$

where $\mu(x, t)$ is defined in (2.2). If we take $\epsilon$ to be a small (fixed) positive value, we then have that

$$
\begin{aligned}
\theta(t) & \approx-\int_{x^{*}-\epsilon}^{x^{*}+\epsilon} \frac{\beta}{(T-t)\left[1+\frac{\mu^{2}}{4 p \beta}\right]} d x \\
& \approx-\frac{\beta[\alpha-\log (T-t)]^{1 / 2}}{(T-t)^{1 / 2}}{ }_{-\epsilon[\alpha-\log (T-t)]^{-1 / 2}(T-t)^{-1 / 2}} \int_{-\log (T-t)]^{-1 / 2}(T-t)^{-1 / 2}}^{\frac{d y}{1+\frac{y^{2}}{4 p \beta}} .}
\end{aligned}
$$

As $t \rightarrow T$ the limits in the above integral tend to $\pm \infty$ so that

$$
\begin{aligned}
\theta(t) & \approx-\frac{\beta[\alpha-\log (T-t)]^{1 / 2}}{(T-t)^{1 / 2}} \int_{-\infty}^{\infty} \frac{d y}{1+\frac{y^{2}}{4 p \beta}} \\
& \approx-\frac{\pi \beta \sqrt{4 p \beta}[\alpha-\log (T-t)]^{1 / 2}}{(T-t)^{1 / 2}} .
\end{aligned}
$$

The contribution to $\theta(t)$ from the range outside that considered will be of $O(1)$ in time and will be asymptotically dominated by the estimate in (4.7). It then follows that within the blow-up region (4.1) reads as

$$
-\tau \dot{x}_{\xi}=\frac{\beta}{(T-t)\left(1+\frac{\mu^{2}}{4 p \beta}\right)} x_{\xi}-\frac{\pi \beta \sqrt{4 p \beta}[\alpha-\log (T-t)]^{1 / 2}}{(T-t)^{1 / 2}} .
$$

The definition of $\mu(x, t)$ in (2.2) suggests an ansatz for the mesh behaviour of the form

$$
x(\xi, t)=x^{*}+(T-t)^{1 / 2}[\alpha-\log (T-t)]^{1 / 2} z(\xi) .
$$

Substituting this into (4.8) gives

$$
-\tau z_{\xi}\left[-\frac{1}{2}+O\left(\frac{1}{\log (T-t)}\right)\right]=\frac{\beta z_{\xi}}{1+\frac{z^{2}}{4 p \beta}}-\pi \beta \sqrt{4 p \beta} .
$$

The choice of the monitor function to be $u^{p-1}$ ensures that the left-hand side and the first term of the right-hand side of (4.1) have the same scale in $(T-t)$. The term $O\left(\frac{1}{\log (T-t)}\right)$ on the left-hand side of (4.10) emphasizes the difference between an exactly self-similar and 
an approximately self-similar solution. Since $z(\xi)$ should be finite in the blow-up region, the left-hand side term is small compared with the first term on the right-hand side if $\tau<<1$ and therefore can be dropped. Hence, to the approximation of order $\tau$ we obtain an asymptotic equation within the blow-up region as

$$
\frac{\beta z_{\xi}}{1+\frac{z^{2}}{4 p \beta}}-\pi \beta \sqrt{4 p \beta}=0
$$

or

$$
\beta \sqrt{4 p \beta} \tan ^{-1}\left(\frac{z}{2 \sqrt{p \beta}}\right)=\pi \beta \sqrt{4 p \beta}\left(\xi-\xi^{*}\right)
$$

where we have assumed that $z=0$ when $\xi=\xi^{*}$, i.e., blow-up occurs at the point $x^{*}=$ $x\left(\xi^{*}, T\right)$.

Equation (4.12) describes the distribution of the mesh points within the peak, and the boundary conditions (4.3) determine $\xi^{*}$. From (4.9) these two conditions correspond in the limit of large $|z|$, i.e.,

$$
\begin{cases}z \rightarrow-\infty, & \text { as } \xi \rightarrow 0 \\ z \rightarrow+\infty, & \text { as } \xi \rightarrow 1\end{cases}
$$

implying

$$
\xi^{*}=\frac{1}{2}
$$

Our choice of $M$ implies that the terms of order $\tau$ will be consistently small inside the blow-up peak.

Combining these results, we deduce that the mesh function $x$ is given by

$$
x(\xi, t)=x^{*}+2 \sqrt{\beta p}(T-t)^{1 / 2}[\alpha-\log (T-t)]^{1 / 2} \tan \left(\pi\left(\xi-\frac{1}{2}\right)\right)+O(\tau)
$$

so that

$$
\frac{x(\xi, t)-x^{*}}{(T-t)^{1 / 2}[\alpha-\log (T-t)]^{1 / 2} \tan \pi\left(\xi-\frac{1}{2}\right)} \rightarrow 2 \sqrt{\beta p} \text { as } t \rightarrow T .
$$

Substituting this into the expression for $u(x, t)$ gives

$$
u(\xi, t)=(T-t)^{-\beta} \beta^{\beta}[\cos (\pi(\xi-1 / 2))]^{2 \beta} .
$$

This expression for $u$ in the computational domain may be easily checked against numerical calculations.

The above analysis only applies for mesh points within the peak. However, as $\xi \rightarrow 0$ or $\xi \rightarrow 1$ the mesh points must eventually tend to 0 and 1 , respectively, and close to $\xi=0$ they correspond to points where $u(x, t)$ is not changing rapidly and hence is bounded as $t \rightarrow T$. If we fix $x$, then as $t \rightarrow T$,

$$
u(\xi, t) \rightarrow\left[\frac{8 \beta^{2} p|\log | x-x^{*}||}{\left(x-x^{*}\right)^{2}}\right]^{\beta}+O\left(x-x^{*}\right)^{2}
$$


Thus,

$$
\int_{0}^{x} u^{1 / \beta} \approx 8 \beta^{2} p \int_{0}^{x} \frac{|\log | x-x^{*}||}{\left(x-x^{*}\right)^{2}} \approx 8 \beta^{2} p \frac{|\log | x-x^{*}||}{\left(x-x^{*}\right)^{2}}
$$

Similarly, from (4.11),

$$
\int_{0}^{1} u^{1 / \beta}=\theta(t) \approx \frac{\beta \pi \sqrt{4 p \beta}[\alpha-\log (T-t)]^{1 / 2}}{(T-t)^{1 / 2}}
$$

so that an equidistributed mesh will give

$$
\int_{0}^{x} u^{1 / \beta} \approx \theta(t) \xi
$$

and hence

$$
8 \beta^{2} p \frac{|\log | x-x^{*}||}{\left(x-x^{*}\right)^{2}} \approx \frac{\beta \pi \sqrt{4 p \beta}[\alpha-\log (T-t)]^{1 / 2} \xi}{(T-t)^{1 / 2}} .
$$

After some manipulation we find that this is precisely the form that (4.16) takes as $\xi \rightarrow 0$ (or indeed as $\xi \rightarrow 1$ ).

We observe from (4.15) and (4.22) that $x$ is close to $x^{*}$ if $\xi$ lies in the interval $[\epsilon, 1-\epsilon]$ where $\epsilon=(T-t)^{1 / 2}[\alpha-\log (T-t)]^{1 / 2}$. Outside this interval, $x$ rapidly tends to 0 or 1 . The implications of this analysis are that if $\Delta \xi$ is the mesh spacing in the computational domain, then a significant number of mesh points will be away from the peak only if

$$
\Delta \xi<(T-t)^{1 / 2}[\alpha-\log (T-t)]^{1 / 2} .
$$

A similar calculation can be made for the arclength monitor function

$$
M=\sqrt{1+u_{x}^{2}} .
$$

Indeed, when $u_{x}$ is large, $M \approx u_{x}$, and we have from (4.1), (4.4), and (4.9) that

$$
\begin{aligned}
& -\tau z_{\xi}\left[-\frac{1}{2}+O\left(\frac{1}{\log (T-t)}\right)\right][\alpha-\log (T-t)]^{1 / 2}(T-t)^{\beta-1 / 2} \\
& =\frac{2 \beta^{\beta+1}|z| z_{\xi}}{4 p \beta\left(1+\frac{z^{2}}{4 p \beta}\right)^{\beta+1}}+\Theta
\end{aligned}
$$

where $\Theta$ is a constant. Thus, when $\tau[\alpha-\log (T-t)]^{1 / 2}(T-t)^{\beta-1 / 2}<<1$, for which we require $p<3$ and $\tau$ to be fixed and small, then within the blow-up region the mesh equation is asymptotic to

$$
\frac{2 \beta^{\beta+1}|z| z_{\xi}}{4 p \beta\left(1+\frac{z^{2}}{4 p \beta}\right)^{\beta+1}}+\Theta=0
$$

which leads to

$$
u(\xi, t)=(T-t)^{-\beta} \beta^{\beta}\left[1-2\left|\xi-\xi^{*}\right|\right] .
$$

However, when $\tau[\alpha-\log (T-t)]^{1 / 2}(T-t)^{\beta-1 / 2}>>1$, which occurs when $p>3$ and $t$ is sufficiently close to $T$, then the left-hand side of (4.25) dominates over other terms and then there exists no solution satisfying the resulting asymptotic equation and the matching 
conditions (4.13). This implies that the mesh (or the coordinate transformation) cannot take the form (4.9) and hence is not "optimal" when $\tau[\alpha-\log (T-t)]^{1 / 2}(T-t)^{\beta-1 / 2}>>1$. Indeed, in this case the timescale for the mesh evolution is greater than $T-t$ and the mesh ceases to evolve. To be precise, this occurs when

$$
\tau(T-t)^{\beta-1 / 2}=\tau(T-t)^{(3-p) / 2(p-1)}=\tau u_{\max }^{(p-3) / 2}>>1 .
$$

Finally we note that the above analysis is general and can also be applied to MMPDE4. Since the argument is quite similar, we omit it here but refer the reader to the discussion in $\S 3$.

4.2. The analysis of the discrete equations. We now turn our attention to an analysis of the discretizations (3.9) and (3.12) of the PDEs for blow-up and mesh evolution. We show that these equations admit a discrete solution which evolves in an "approximately self-similar" manner very closely related to that of the solution described in $\S 2$. Although we do not prove that this discrete solution is an attractor, the numerical calculations given in $\S 5$ strongly imply that it is, and the asymptotic predictions of this section agree with the observations in $\S 5$. (We emphasize at this point that the numerical solution may have a slightly different blow-up time from the analytical solution. However, as we are interested in the dynamics close to blow-up rather than the blow-up time itself we shall treat the blow-up times of the analytic and numerical methods as the same.)

Since the discrete equations have the same scaling invariance as the continuous ones, it is reasonable to consider a discrete solution in terms of similarity variables closely related to those in (2.6), namely

$$
\left\{\begin{array}{l}
w_{i}=(T-t)^{\beta} u_{i} \\
y_{i}(s)=\left(x_{i}-x^{*}\right)(T-t)^{-1 / 2} \\
s=-\log (T-t)
\end{array}\right.
$$

In these similarity variables we consider solutions with $w_{i}$ independent of time. Substituting into (3.9) gives

$$
\begin{aligned}
& \beta w_{i}+\frac{w_{i+1}-w_{i-1}}{y_{i+1}-y_{i-1}}\left(\frac{1}{2} y_{i}-\dot{y}_{i}\right) \\
& =\frac{2}{y_{i+1}-y_{i-1}}\left(\frac{w_{i+1}-w_{i}}{y_{i+1}-y_{i}}-\frac{w_{i}-w_{i-1}}{y_{i}-y_{i-1}}\right)+w_{i}^{p} .
\end{aligned}
$$

The equation (4.30) is essentially a discretization of (2.7) on the nonuniform mesh $\left\{y_{i}\right\}$ and must also satisfy a discrete form of the boundary conditions (2.8). Like (2.7) it also possesses discrete "similarity solutions" for which both $w_{i}$ and $y_{i}$ are independent of time, and these solutions satisfy a discretization of the steady state of (2.7). However, this latter equation has only one solution which does not grow exponentially with $y$, and that is the constant solution, which does not satisfy the boundary conditions. (We note that $w_{i} \equiv \beta^{\beta}$ is also a solution of (4.30).) Provided that the mesh $\left\{y_{i}\right\}$ is sufficiently fine we will expect that the (nonconstant) discrete solutions of the steady state of (4.30) should approximate their continuous counterparts and also grow rapidly with $i$. Such solutions will not then match the boundary conditions. We can conclude from this reasoning that (4.30) is unlikely to have a steady state solution and that we should instead seek a solution which, like its analytical counterpart, is approximately self-similar rather than self-similar. We can compute these as in $\S 2$ by setting

$$
y_{i}(s)=g(s) z_{i}
$$

where $z_{i}$ is independent of time and the function $g$ satisfies (cf. (2.12))

$$
g(s) \rightarrow \infty \text { and } \frac{g^{\prime}}{g} \rightarrow 0 \text { as } s \rightarrow \infty
$$


Substituting into (4.30) and letting $s \rightarrow \infty$, we have

$$
\beta w_{i}+\frac{w_{i+1}-w_{i-1}}{z_{i+1}-z_{i-1}} \frac{z_{i}}{2}=w_{i}^{p}
$$

This is a centred difference discretization of (2.14), and hence, provided the mesh $\left\{z_{i}\right\}$ is sufficiently regular,

$$
w_{i}=\left(\frac{1}{\beta}+z_{i}^{2}\right)^{-\beta}+\epsilon_{i},
$$

where $\epsilon_{i}$ is a small error which decreases with the number of mesh points. As before, the form of $g$ for large $s$ can be derived from formal scaling arguments which give

$$
g(s)=c[\alpha+s]^{1 / 2}
$$

We can repeat the arguments in [BK88] to deduce the value of the constant $c$ which, to within a small error similar to $\epsilon_{i}$, is consistent with (2.16). This demonstrates that an approximately self-similar solution for the numerical scheme (4.30) exists which has the correct dependence upon $z_{i}$ and hence upon $x_{i}$. Finally, we evaluate $z_{i}$. The moving mesh equation (3.12) gives

$$
\begin{aligned}
& -\tau\left(\dot{x}_{i+1}-2 \dot{x}_{i}+\dot{x}_{i-1}\right) \\
& =\frac{1}{2}\left(u_{i+1}^{p-1}+u_{i}^{p-1}\right)\left(x_{i+1}-x_{i}\right)-\frac{1}{2}\left(u_{i}^{p-1}+u_{i-1}^{p-1}\right)\left(x_{i}-x_{i-1}\right),
\end{aligned}
$$

which has first integral

$$
-\tau\left(\dot{x}_{i+1}-\dot{x}_{i}\right)=\frac{1}{2}\left(u_{i+1}^{p-1}+u_{i}^{p-1}\right)\left(x_{i+1}-x_{i}\right)+\theta(t) .
$$

Substituting for $z_{i}$ and $w_{i}$ we have after some manipulation that

$$
\begin{aligned}
& \frac{\tau}{2}\left(z_{i+1}-z_{i}\right)\left(1+O\left(\frac{1}{s}\right)\right) \\
& =\frac{1}{2}\left(w_{i+1}^{p-1}+w_{i}^{p-1}\right)\left(z_{i+1}-z_{i}\right)+\frac{(T-t)^{1 / 2}}{g(s)} \theta(t) .
\end{aligned}
$$

Choosing $\theta$ appropriately and using (4.34), this is a centred difference discretization of (4.10), and hence $z_{i}$ is a discrete approximation to $z(\xi)$ given in (4.9).

We conclude that the discrete form of the PDE coupled with MMPDE6 admits an approximately self-similar solution for which both the mesh $x_{i}$ and the function $u_{i}$ are consistent approximations to the mesh $x(\xi, t)$ and function $u(\xi, t)$ calculated in $\S 4$.1. Provided that this solution is (like its analytic counterpart) an attractor for a wide class of initial data, then the numerical method given will faithfully reproduce the dynamics of the blow-up peak for all times up to the blow-up time. In particular, the asymptotic estimates given in $\S 4.1$ will be reproduced in the numerical calculations. It is difficult to analyze analytically whether the solution is an attractor, and we do not attempt it here.

5. Numerical examples. We now present some numerical calculations for several blowup problems using MMPDE4 and MMPDE6. These calculations support the analysis of $\S \S 3$ and 4 and also illustrate the effect of smoothing the monitor function $M$. For these calculations we take $u_{0}(x)=20 \sin (\pi x)$.

After spatial discretization, the resulting ODE systems are solved using the double precision version of the stiff ODE solver DASSL [Pet82]. The time integration uses the backward differentiation formulas (BDF), wherein an approximate Jacobian is computed by DASSL internally using finite differences. The relative and absolute local time-stepping error tolerances 

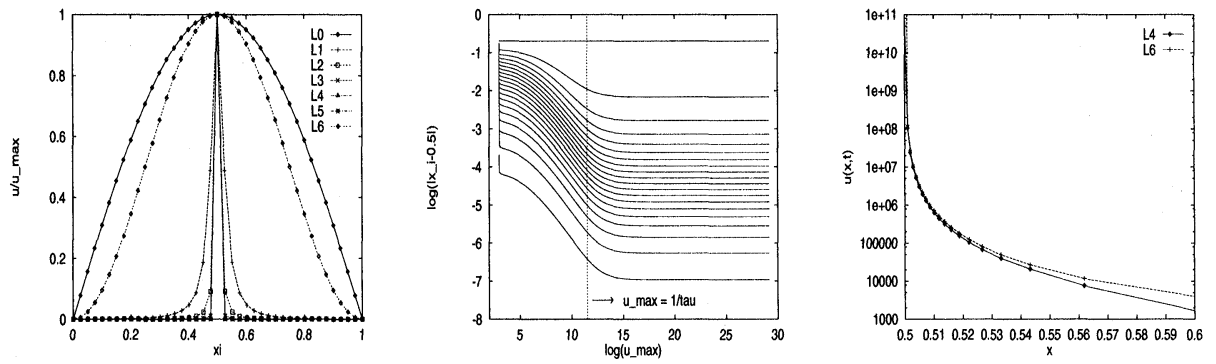

FIG. 1 (1a, 1b, and 1c from the left). MMPDE4 without smoothing is used for solving (2.1) with $p=2$. In Figs. 1a and 1c L0 : initial solution, $L 1: u_{\max }=10^{8}, L 2: u_{\max }=10^{9}, L 3: u_{\max }=10^{10}, L 4: u_{\max }=10^{11}$, L5: $u_{\max }=5 \times 10^{11}$, L6: the asymptotic solution.

(in a root-mean-square norm) are chosen as rtol $=10^{-8}$ and atol $=10^{-8}$, respectively. Unless stated otherwise, we use a uniform mesh (in $x$ ) initially with $N=40$ and take $\tau=10^{-5}$. As pointed out in [HRR94a] and [HRR94b], the choice of value for the time correction parameter $\tau$ is not critical and can generally be fixed to be a small positive value.

5.1. Calculations using MMPDE4. The analysis in $\S 3$ indicates that MMPDE4 ceases to evolve the mesh when the timescale of the blow-up is less than $\tau$, i.e., when

$$
(T-t)<\tau
$$

Since $u\left(x^{*}, t\right) \approx(T-t)^{-\beta}$, the mesh ceases to evolve when

$$
u\left(x^{*}, t\right)>\tau^{-\beta},
$$

after which MMPDE4 gives a nonuniform but essentially fixed mesh. The results of [AB94] show that if $p>2, u_{i}$ will blow up at only one point and if $p=2$ it will blow up at three points, but the blow-up is asymptotically dominated by the growth at one point.

To confirm these results we integrate (2.1) when $p=2$ coupled with MMPDE4 using the monitor function given by (3.6). Blow-up occurs at $T=0.082291$ and $x^{*}=\frac{1}{2}$ so that the maximum value occurs at $u_{20}$. In Fig. 1a we present a graph of the scaled discrete solution values $\left\{\frac{u_{i}}{u_{20}}\right\}$ for $u_{20}=10^{8}, 10^{9}, 10^{10}, 10^{11}, 5 \times 10^{11}$, and in Fig. $1 \mathrm{~b}$ we show the corresponding mesh, plotting $\log \left|x_{i}-\frac{1}{2}\right|$ against $\log \left(u_{20}\right)$. From the second figure it is clear that the mesh evolves until $u_{20} \approx \frac{1}{\tau}$ and is then fixed. The results of Fig. 1a show convergence of the normalized solution to a delta function, demonstrating that, effectively, it is only $u_{20}$ which is blowing up. In Fig. 1c we plot the approximation for $u(x, t)$ when $u_{20}=10^{11}$ and compare it with the asymptotic function $u(x, t)$ given in (2.3). It is clear that it approximates the limiting asymptotic function rather well (except at the blow-up point), showing that the mesh generated by MMPDE4 leads to an accurate approximation to $u(x, t)$ away from the point of blow-up and the behaviour of $u(x, T)$ is well approximated in this range. Indeed, at the point when MMPDE4 ceases to evolve the mesh, the mesh point nearest $\frac{1}{2}$ is given by

$$
x_{i}=\frac{1}{2}+O\left(\Delta \xi \tau^{1 / 2} \log ^{1 / 2} \tau\right)
$$

where $\Delta \xi$ is the mesh spacing in the computational domain. Thus MMPDE4 gives an accurate picture of the evolution of the solution in the region $\left[0, \frac{1}{2}-O\left(\Delta \xi \tau^{1 / 2} \log ^{1 / 2} \tau\right)\right] \cup\left[\frac{1}{2}+\right.$ $\left.O\left(\Delta \xi \tau^{1 / 2} \log ^{1 / 2} \tau\right), 1\right]$, but it will not resolve the structure in the remaining blow-up region. 

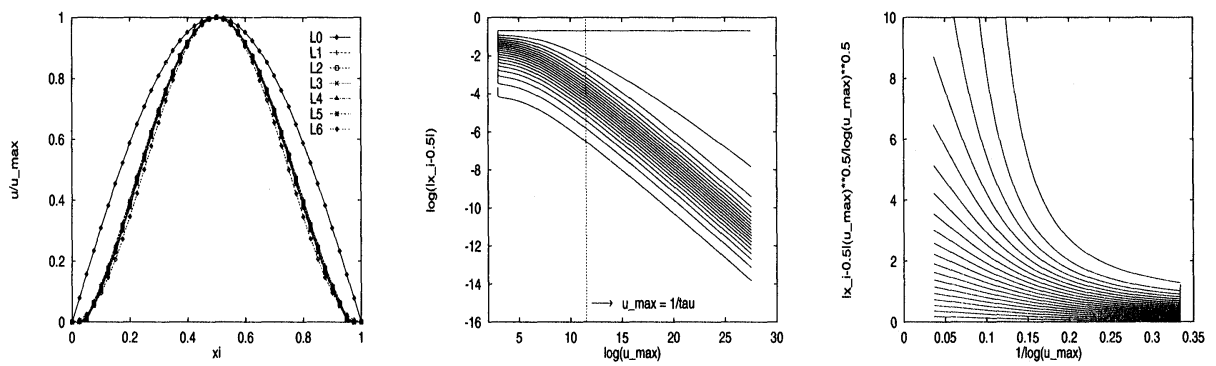

FIG. 2 (2a, 2b, and 2c from the left). MMPDE6 without smoothing is used for solving (2.1) with $p=2$. In Fig. $2 \mathrm{a}$ L0: initial solution, $L 1: u_{\max }=10^{8}, L 2: u_{\max }=10^{9}, L 3: u_{\max }=10^{10}, L 4: u_{\max }=10^{11}, L 5: u_{\max }=5 \times 10^{11}$, L6: the asymptotic solution.

5.2. Calculations using MMPDE6. The analysis given in $\S \S 3$ and 4 indicates that (in contrast to MMPDE4) using MMPDE6 with the monitor function given by (3.6) should give an accurate resolution of the blow-up peak. A calculation of the evolution of the PDE gives a blow-up time of $T=0.082283$ when $p=2$. The scaled solution $\left\{\frac{u_{i}}{u_{20}}\right\}$ for $u_{20}=10^{8}, 10^{9}, 10^{10}, 10^{11}, 5 \times 10^{11}$, is given in Fig. 2a together with the theoretical scaled solution $\cos ^{2} \pi\left(\xi-\frac{1}{2}\right)$ where $\xi=i \Delta \xi$ (cf. (2.10) and (4.17)). It is clear from the figure that there is close agreement between the predicted and computed curves implying that the solution calculated in $\S 4.2$ is indeed an attractor. Figure $2 \mathrm{~b}$ shows a graph of $\log \left|x_{i}-\frac{1}{2}\right|$ as a function of $\log u_{\max }$ with clear evidence that each of the mesh points (apart from those close to the boundary) evolves in the same (approximately self-similar) manner. To confirm that the mesh behaves precisely as predicted in $\S 4$, we give a graph in Fig. $2 \mathrm{c}$ of $\frac{\left|x_{i}-\frac{1}{2}\right|}{(T-t)^{1 / 2}|\log (T-t)|^{1 / 2}}$ as a function of $|\log (T-t)|^{-1}$, where we use the estimate $T-t=\frac{1}{u_{20}}$. According to formula (4.16),

$$
\frac{\left|x_{i}-\frac{1}{2}\right|}{(T-t)^{1 / 2}|\log (T-t)|^{1 / 2}} \approx \sqrt{8} \tan \pi\left(\xi_{i}-\frac{1}{2}\right)\left[1-\frac{1}{2} \frac{\alpha}{|\log (T-t)|}\right]
$$

as $|\log (T-t)| \rightarrow \infty$. Thus, for each $i$ we expect to obtain a graph which is asymptotically linear in $|\log (T-t)|^{-1}$ and converges to $\sqrt{8} \tan \pi\left(\xi_{i}-\frac{1}{2}\right)$ with slope $-\frac{1}{2} \alpha \sqrt{8} \tan \pi\left(\xi_{i}-\frac{1}{2}\right)$. The figure clearly shows that this is the case, and we estimate $\alpha$ to be 7 .

Thus MMPDE6 accurately reproduces the evolution of the peak profile; however, with 40 mesh points $x_{39}-\frac{1}{2} \approx 35.94(T-t)^{1 / 2}|\log (T-t)|^{1 / 2}$ so that $x_{39}=0.500966$ when $u_{20}=10^{11}$. Thus, the mesh points are concentrated in the blow-up region, so that the solution away from the peak is poorly resolved.

If smoothing as described in $\S 3$ is introduced, the resulting form of $\frac{u_{i}}{u_{20}}$ for the same value of $u_{\max }$ is given in Fig. 3a. In this case, the blow-up is slightly delayed to $T=0.082319$. Now, the scaled discrete solution $\left\{\frac{u_{i}}{u_{20}}\right\}$ no longer approaches a constant curve, but instead, slowly approaches the delta function which would be obtained using a uniform mesh. This is because smoothing tends to place fewer points in the peak and more points away from the peak. Nevertheless, we see from Fig. $3 b$ that the mesh points close to the peak evolve in a manner similar to those in Fig. 2b. Hence, whilst these points are close to the blow-up point, the analysis of $\S 4$ still applies and the value of $u_{i}$ will be close to $u\left(x_{i}, t\right)$. Thus, although smoothing does not precisely align the mesh along the level curves of the function $\mu$ in (4.16), it still gives an accurate resolution of the structure of the peak.

The resolution of the peak in the two cases is made apparent in Figs. $4 \mathrm{a}$ and $4 \mathrm{~b}$, which plot $\frac{u_{i}}{u_{20}}$ as a function of $\log \left|x_{i}-\frac{1}{2}\right|$ for $u_{20}=10^{9}, 10^{10}, 10^{11}, 5 \times 10^{11}$ for the unsmoothed 

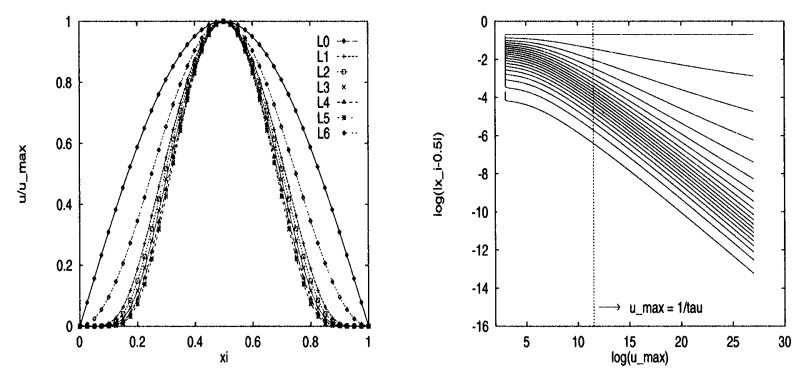

FIG. 3 (3a and 3b from the left). MMPDE6 with smoothing $\left(i_{p}=2\right)$ is used for solving $(2.1)$ with $p=2$. In Fig. 3a L0: initial solution, $L 1: u_{\max }=10^{8}, L 2: u_{\max }=10^{9}, L 3: u_{\max }=10^{10}, L 4: u_{\max }=10^{11}$, $L 5: u_{\max }=5 \times 10^{11}$, L6: the asymptotic solution which is valid only for the unsmoothed case.
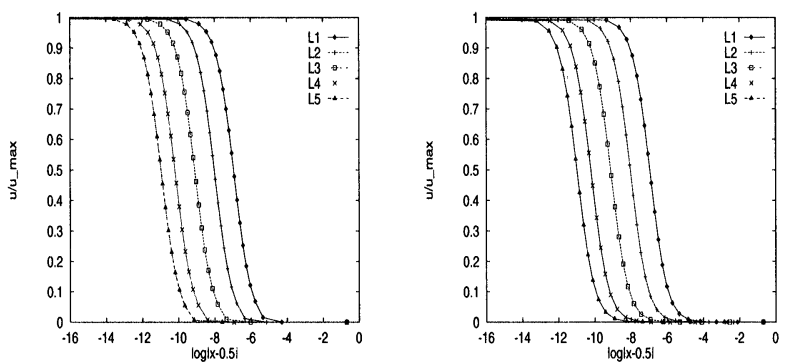

FIG. 4 (4a and $4 \mathrm{~b}$ from the left). MMPDE6 is used for solving (2.1) with $p=2$. Fig. $4 \mathrm{a}$ is for the case without smoothing and Fig. $4 \mathrm{~b}$ for that with smoothing $\left(i_{p}=2\right)$. In the figures, $L 1: u_{\max }=10^{8}, L 2: u_{\max }=10^{9}$, $L 3: u_{\max }=10^{10}, L 4: u_{\max }=10^{11}, L 5: u_{\max }=5 \times 10^{11}$.

and smoothed meshes, respectively. The coordinate $\log \left|x_{i}-\frac{1}{2}\right|$ is used to stretch the length scale close to the peak so that its structure can be seen more easily. The two figures are almost identical, confirming that smoothing does not affect the accuracy of the resolution of the peak for these values of $u$ (although presumably it would for much larger values). As a further test of the accuracy of the schemes the graphs in Figs. $4 \mathrm{a}$ and $4 \mathrm{~b}$ can be compared with the graph of the function

$$
\frac{u}{u_{\max }}=\left[1+\frac{\left(x-\frac{1}{2}\right)^{2}}{8(T-t)(\alpha-\log (T-t))}\right]^{-1}
$$

where $u_{\max } \equiv(T-t)^{-1}$. Indeed, the value of $\alpha=7$ estimated earlier gives a good fit for each of the curves.

Although smoothing is not necessary when $p=2$, it becomes essential when dealing with more severe nonlinearities, as unsmoothed moving mesh equations can tend to introduce oscillations in solutions away from singularities by placing too few points there (e.g., see [VBFZ89]). We see this by taking $p=5, M=u^{4}$, and using an initial mesh equidistributed with respect to the initial solution $u(x, 0)=20 \sin (\pi x)$. Figure 5a shows the computed value of $\left(\frac{u_{i}}{u_{\max }}\right)^{4}$ plotted as a function of $\xi$ in the case of no smoothing for $u_{\max }^{5}=10^{8}, 10^{9}, 10^{10}, 10^{11}$, and $10^{12}$. These computed solutions approximate $\cos ^{2} \pi\left(\xi-\frac{1}{2}\right)$ for $u_{\max }^{5}=10^{11}$, but deviate from it, due to mesh oscillations, when $u_{\max }^{5}>10^{11}$. In contrast, the smoothed solution (obtained with $i_{p}=4$ ) does not oscillate and still retains enough points near the blow-up point to give good resolution in the peak, where for reference purposes we also give the asymptotic solution $\cos ^{2} \pi\left(\xi-\frac{1}{2}\right)$ which applies only for the nonsmooth moving mesh case (see Fig. 5b). 

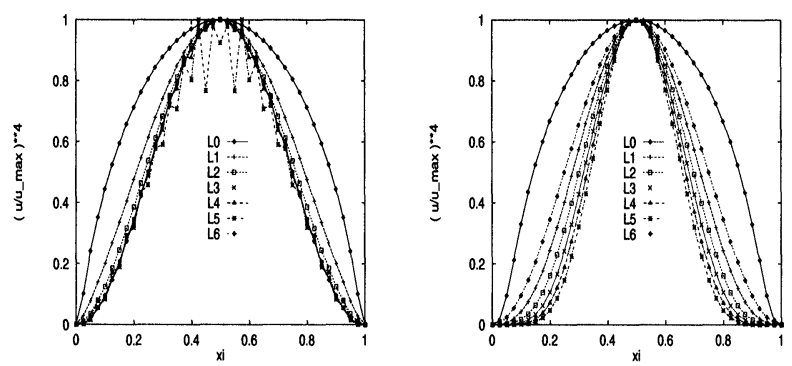

Fig. 5 (5a and $5 \mathrm{~b}$ from the left). MMPDE6 is used for solving (2.1) with $p=5$. In Fig. 5a (without smoothing), L0: initial solution, $L 1: u_{\max }^{5}=10^{8}, L 2: u_{\max }^{5}=10^{9}, L 3: u_{\max }^{5}=10^{10}, L 4: u_{\max }^{5}=10^{11}, L 5: u_{\max }^{5}=10^{12}$, L6: the asymptotic solution. In Fig. $5 \mathrm{~b}$ (with smoothing $\left(i_{p}=4\right)$ ), L0: initial solution, $L 1: u_{\max }^{5}=10^{9}$, $L 2: u_{\max }^{5}=10^{10}, L 3: u_{\max }^{5}=10^{11}, L 4: u_{\max }^{5}=10^{12}, L 5: u_{\max }^{5}=10^{13}, L 6:$ the asymptotic solution which is valid only for the unsmoothed case.
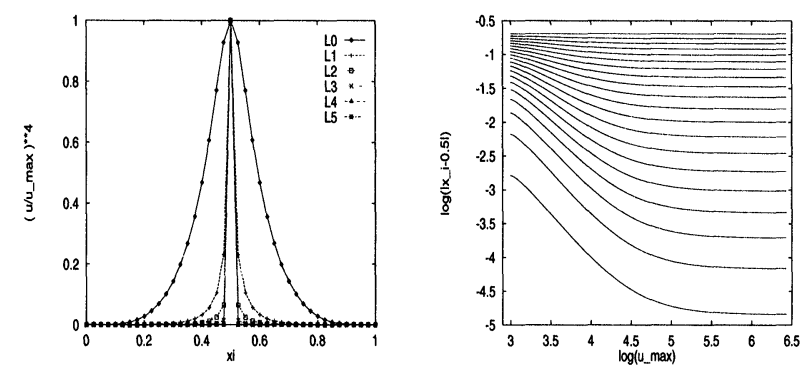

FIG. $6\left(6 \mathrm{a}\right.$ and $6 \mathrm{~b}$ from the left). MMPDE6 with smoothing $\left(i_{p}=4\right)$ and the arclength monitor function is used for solving (2.1) with $p=5$. In Fig. $6 \mathrm{a}, L 0$ : initial solution, $L 1: u_{\max }^{5}=10^{10}, L 2: u_{\max }^{5}=10^{11}, L 3: u_{\max }^{5}=10^{12}$, $L 4: u_{\max }^{5}=10^{13}, L 5: u_{\max }^{5}=10^{14}, L 6:$ the asymptotic solution.

It is interesting to repeat the calculations for $p=5$ using the common choice of the arclength monitor function $M=\sqrt{1+u_{x}^{2}}$ [HRR94b]. The results of $\S 4$ indicate that the mesh will cease to evolve when $\tau u_{\max }^{(p-3) / 2}>>1$, which in this case occurs when $u_{\max }>>1 / \tau$. In Figs. $6 \mathrm{a}$ and $6 \mathrm{~b}$ we present the resulting computed solution for different values of $u_{\max }^{5}$ and the evolution of the mesh (with smoothing $i_{p}=4$ ), respectively. We see clearly that the mesh ceases to evolve in this case. The value of $u_{\max }$ at which this occurs is, in fact, rather smaller than the value predicted above. This is a result of the smoothing we have employed.

5.3. Exponential nonlinearities. We can easily extend the results of the previous subsections to the case of an exponential nonlinearity, such as for the equation

$$
u_{t}=u_{x x}+e^{u}
$$

and its generalization

$$
u_{t}=\left(\left|u_{x}\right|^{\sigma} u_{x}\right)_{x}+e^{u}
$$

using the monitor function $M(u)=e^{u}$. It is well known [Dol85] that close to the blow-up point the solutions of (5.6) have an approximately self-similar solution of the form

$$
u(x, t) \sim-\log (T-t)-\log \left(1+\frac{x^{2}}{4(T-t) \log (T-t)}\right) .
$$


The solutions of (5.7) are, in contrast, self-similar such that

$$
u(x, t) \sim-\log (T-t)+W\left(\frac{x}{(T-t)^{1 /(\sigma+2)}}\right),
$$

where for small $\sigma$ the function $W$ has the form

$$
W(\zeta)=-\log \left(1+\frac{1}{2}\left(\frac{\sigma}{2}\right)^{1-\sigma} \zeta^{2}\right)
$$

(see [BG94]). Using an analysis identical to that given earlier we may derive the following formal result.

PROPOSITION 5.1. Let $M(u)=e^{u}$.

(i) If $\sigma=0$, then an equidistributed mesh transformation is given by

$$
\begin{gathered}
x(\xi, t)=x^{*}+(4(T-t) \log (T-t))^{1 / 2} \tan ^{-1}\left(\pi\left(\xi-\frac{1}{2}\right)\right), \\
\frac{e^{u(\xi, t)}}{e^{u\left(\frac{1}{2}, t\right)}} \rightarrow \cos ^{2} \pi\left(\xi-\frac{1}{2}\right) .
\end{gathered}
$$

(ii) Let

$$
f(x) \equiv \int_{0}^{x} \frac{d x}{1+y^{\sigma+2}}, \quad F \equiv 2 f(\infty)
$$

If $\sigma>0, \sigma$ small, then

$$
\begin{gathered}
x(\xi, t)=x^{*}+\left(2(T-t)\left(\frac{2}{\sigma}\right)^{1-\sigma}\right)^{1 /(\sigma+2)} f^{-1}\left[F\left(\xi-\frac{1}{2}\right)\right], \\
\frac{e^{u(\xi, t)}}{e^{u\left(\frac{1}{2}, t\right)}} \rightarrow \frac{1}{1+\left(f^{-1}\left[F\left(\xi-\frac{1}{2}\right)\right]\right)^{2}} .
\end{gathered}
$$

Computations with the exponential nonlinearity raise issues similar to those discussed earlier, especially with regard to smoothing. For example, if $\sigma=0$ then a calculation with $u_{0}(x)=5 \sin (\pi x)$ and no smoothing is successful when $u_{\max }<15\left(e^{u_{\max }}<3 \times 10^{6}\right)$, giving a close approximation to the asymptotic results, but it becomes unstable for larger values of $u$. We illustrate this in Fig. 7a, giving $e^{u_{i}-u_{\max }}$ for $u_{\max }=10,11.6,13.33,15,16.6$. A graph of the resulting mesh in Fig. $7 \mathrm{~b}$ shows the oscillations in the mesh points at the boundary even more dramatically. In Figs. $7 \mathrm{c}$ and $7 \mathrm{~d}$ we give the corresponding results with smoothing, taking $i_{p}=4$.

It is interesting to compare the functions $u(x, t)$ computed in these cases, and in Figs. $7 \mathrm{e}$ and $7 \mathrm{f}$ we present graphs of $e^{u-u_{\max }}$ as a function of the physical coordinate $x$ for $u_{\max }=$ $10,11.6,13.33,15,16.6$ for both the unsmoothed and smoothed cases, respectively. These graphs are nearly identical, except that the peak is smoother in Fig. 7f. Figure 8 gives the results of the same calculation on a fixed, uniform mesh. We conclude again that smoothing greatly improves the method without losing the advantages of mesh adaption. 

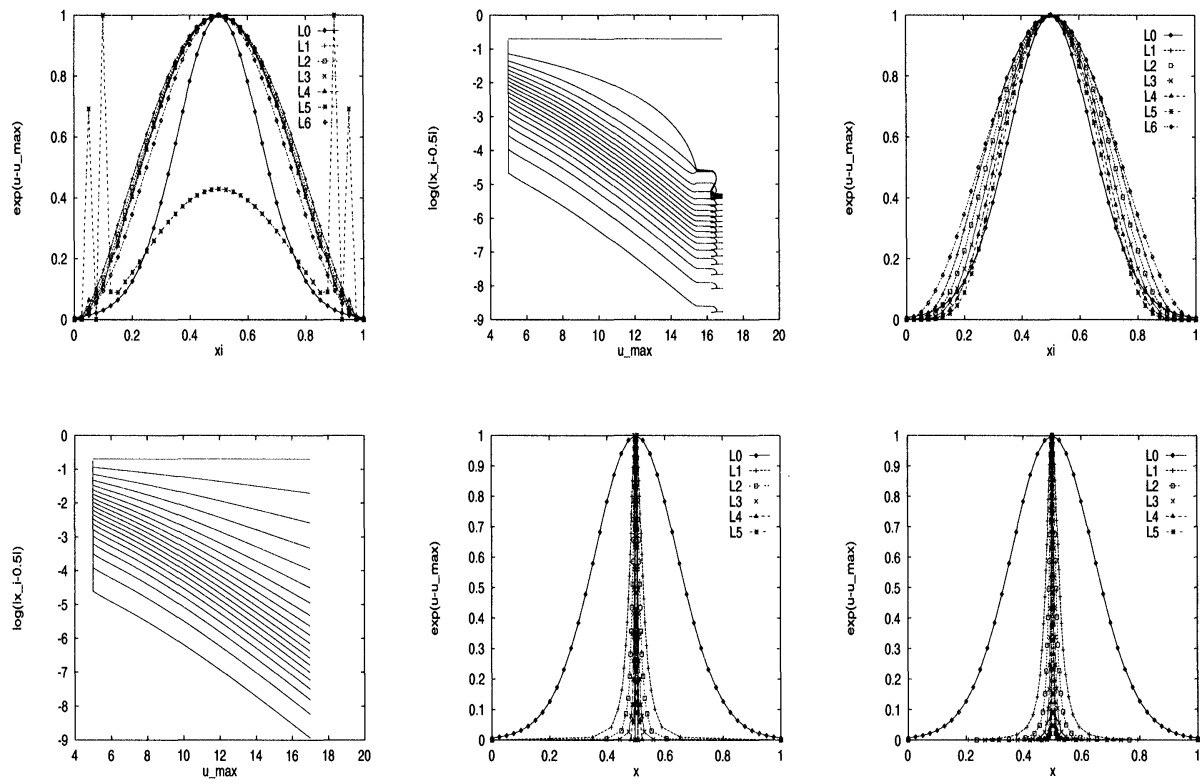

Fig. 7 (7a, 7b, 7c, 7d, 7e, and 7f from the top left). MMPDE6 is used for solving (5.6). Figures 7a, 7b, and 7e are for the case without smoothing and Figs. $7 \mathrm{c}, 7 \mathrm{~d}$, and $7 \mathrm{f}$ are for that with smoothing $\left(i_{p}=4\right)$. In the figures, L0: initial solution, $L 1: u_{\max }=10, L 2: u_{\max }=11.6, L 3: u_{\max }=13.33, L 4: u_{\max }=15, L 5: u_{\max }=16.6, L 6:$ the asymptotic solution which is valid only for the unsmoothed case.

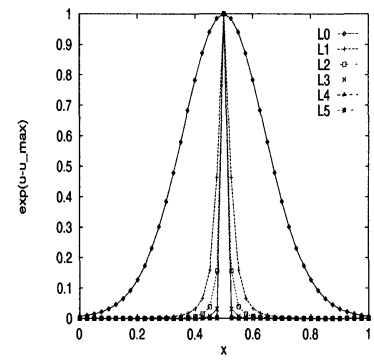

FIG. 8. A fixed, uniform mesh is used for solving (5.6). L0: initial solution, $L 1: u_{\max }=10, L 2: u_{\max }=11.6$, $L 3: u_{\max }=13.33, L 4: u_{\max }=15, L 5: u_{\max }=16.6$.

5.4. Degenerate parabolic equations. To show the ability of the MMPDE methods to handle different types of blow-up, we conclude this section with the application of the MMPDE methods to a problem that has not been as extensively analyzed as (2.1) or (2.20). We consider the degenerate parabolic problem

$$
x u_{t}=u_{x x}+15 u^{2}
$$

subject to the boundary conditions $u(0, t)=u(1, t)=0$. This problem has been studied theoretically and numerically in [Flo91] and [SF90]. It is shown in [Flo91] that a distinct feature of the blow-up for (5.16) from that for nondegenerate problems (e.g., (1.1)) is that the solution blows up at the boundary $x=0$. In contrast to that for nondegenerate problems, the blow-up behaviour for degenerate problems like (5.16) is as yet not well understood.

The results obtained with MMPDE6 and smoothing $\left(i_{p}=2\right)$ are shown in Figs. 9a-9e. In the computation, we have used $u_{0}(x)=4 x(1-x)$ and $M=u^{3 / 2}$, which is chosen so that the 

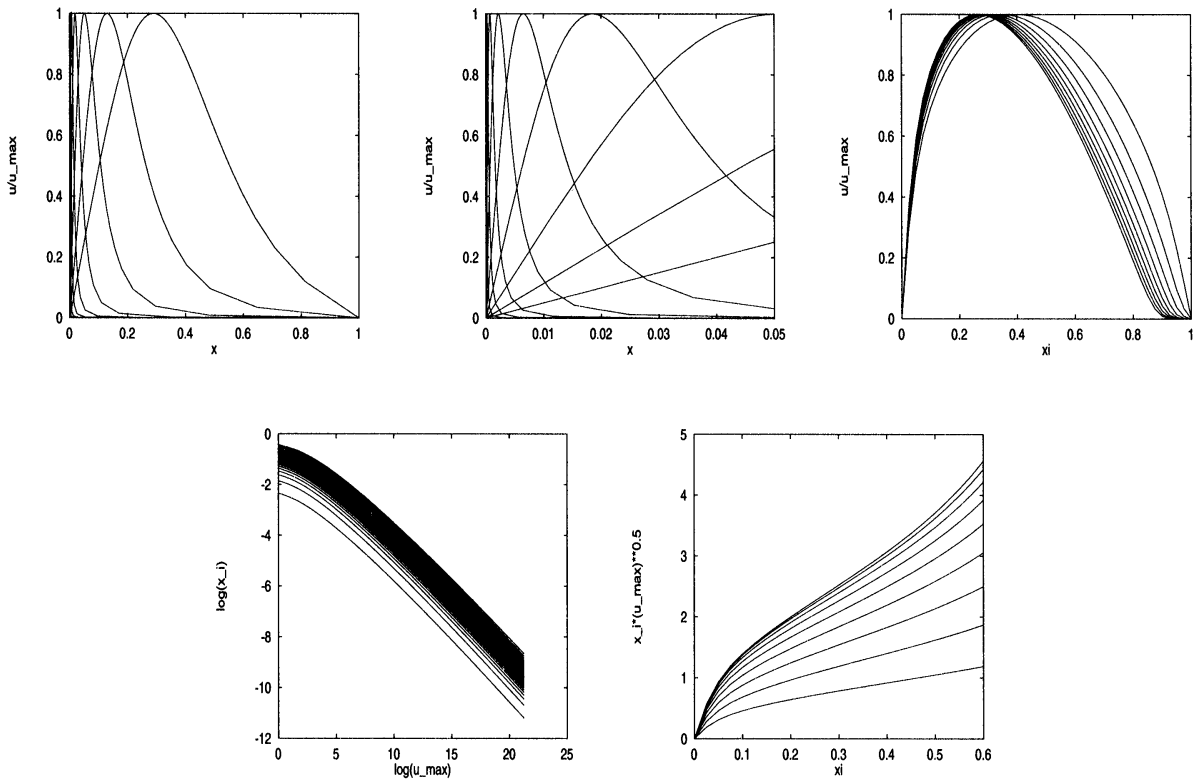

FIG. 9 (9a, 9b, 9c, 9d, and 9e from the top left). MMPDE6 with smoothing $\left(i_{p}=2\right)$ is used for solving (5.16). Figure $9 \mathrm{~b}$ is the magnification of the left portion of Fig. 9a.

extended system consisting of the physical PDE and the MMPDE preserves the underlying scaling invariance of (5.16). The results show the blow-up at the boundary $x=0$. Our computation gives a blow-up time of $T \approx 0.056015$, which is close to the value obtained in [SF90] with a so-called peak-tracking strategy. The computation required roughly 36.7 seconds of CPU time on an SGI R3000 Indigo.

6. Conclusions and comments. In this paper we have considered PDEs which model blow-up problems for which scaling invariance plays a natural role in describing the underlying solution structures. When one computes the solutions to such problems, adaptive mesh methods are virtually unavoidable. It is useful to interpret such numerical methods as discretizations (on a uniform mesh) of the PDEs rewritten in terms of a computational coordinate transformation. This transformation can in turn be defined through an MMPDE [HRR94b] which is determined by equidistribution with respect to a monitor function $M$. It is natural to seek a monitor function which preserves the scaling invariance; note that this does not require detailed knowledge of the solution behaviour itself. The scaling invariance is then preserved by the discretization, i.e., by the actual moving mesh method which is implemented. To our knowledge, this is the first instance in which rigorous analysis has been used to motivate the choice of specific monitor functions. For the blow-up problems with known detailed solution behaviour, the solutions are only approximate similarity solutions, and, quite remarkably, their structure is also preserved by the discrete equations when suitable monitor functions are chosen. As a result, with relatively few mesh points the analytic structure of the blow-up solutions can be accurately computed in a very efficient way. We briefly present some computations (without accompanying analysis) for a degenerate parabolic problem whose solution has not been extensively analyzed.

A comparison of our approach for blow-up problems with those of others is outside the scope of this paper. Nevertheless, we have tried to emphasize the naturalness of our method for automatically picking up the self-similar coordinate and to demonstrate the ease with which these problems can often be solved with little a priori knowledge. 
This is not to say that the moving mesh methods are without pitfalls, however. It is important to use proper monitor functions, and examples are given where the popular choice of arclength fails to perform adequately. The MMPDEs must also be implemented with care. For example, in Figs. 1a and 1b MMPDE4 with fixed $\tau$ fails to give the proper blow-up structure when the solution becomes sufficiently large, and the question of whether or not to use mesh smoothing, and if so, the judicious choice of one, are issues with no simple resolution. The blow-up problems considered here are part of a larger framework of PDEs having similarity solutions for which MMPDEs with suitable monitor functions preserve the scaling invariance, and we are in the process of developing a theory for such problems.

\section{REFERENCES}

[AB94] L. ABIA AND C. J. BUDD, Blow-up in uniform discretisations of parabolic PDEs, preprint, 1994.

[BB92] J. BEBERNES AND S. BRICHER, Final time blowup profiles for semilinear parabolic equations via centre manifold theory, SIAM J. Appl. Math., 23 (1992), pp. 852-869.

[BDG93] C. J. BudD, J. W. Dold, AND V. A. GALAKTIONOv, Self-similar solutions of a quasilinear diffusion problem, submitted for publication.

[BDS93] C. J. BUDD, J. W. Dold, AND A. M. STUART, Blowup in a parabolic equation with constraints, SIAM J. Appl. Math., 53 (1993), pp. $718-742$.

[BE89] J. Bebernes AND D. Eberly, Mathematical Problems for Combustion Theory, Appl. Math. Sci., 83, Springer-Verlag, 1989.

[Ber89] M. BERGER, Some applications of adaptive mesh refinement, Adaptive Methods for Partial Differential Equations, J. E. Flaherty, P. J. Paslow, M. S. Shephard, and J. D. Vasilakis, eds., SIAM, Philadelphia, 1989.

[BG94] C. J. Budd AND V. A. GALAKTIONOv, Critical diffusion exponents for blow-up in quasi-linear elliptic PDEs with exponential source, Proc. Roy. Soc. Edinb., to appear.

[BK88] M. BERGER AND R. V. KOHN, A rescaling algorithm for the numerical calculation of blowing-up solutions, Comm. Pure Appl. Math., 41 (1988), pp. 841-863.

[Cho81] A. J. CHORIN, Estimates of intermittency, spectra, and blow-up in developed turbulence, Comm. Pure Appl. Math., 34 (1981), pp. 853-866.

[DD87] E. A. Dorfi AND L. O'C. DRURY, Simple adaptive grids for 1-D initial value problems, J. Comp. Phys., 69 (1987), pp. 175-195.

[Dol85] J. W. DoLD, Analysis of the early stage of thermal runaway, Quart. J. Mech. Appl. Math., 38 (1985), pp. 361-387.

[FK92] S. FILIPPAS AND R. Kohn, Refined asymptotics for the blow up of $u_{t}-\Delta u=u^{p}$, Comm. Pure Appl. Math., 45 (1992), pp. 821-869.

[Flo91] M. S. FLOATER, Blow-up at the boundary for degenerate semilinear parabolic equations, Arch. Rational Mech. Anal., 114 (1991), pp. 57-77.

[FM85] A. FRIEDMAN AND B. MCLEOD, Blow up of positive solutions of semilinear heat equations, Indiana Univ. Math. J., 34 (1985), pp. 425-447.

[FVZ90] R. M. FuRzeland, J. G. VeRwer, AND P. A. Zegeling, A numerical study of three moving grid methods for one-dimensional partial differential equations which are based on the method of lines, J. Comp. Phys., 89 (1990), pp. 349-388.

[Gel63] I. M. Gelfand, Some problems in the theory of quasilinear equations, Amer. Math. Soc. Trans., 29 (1963), pp. 295-381.

[GK85] Y. GIGA AND R. KoHN, Asymptotically self-similar blowup of semilinear heat equations, Comm. Pure Appl. Math., 38 (1985), pp. 297-319.

[GP91] V. A. GALAKTIONOv AND S. A. PosashKov, Single point blow-up for N-dimensional quasilinear equations with gradient diffusion and source, Indiana Univ. Math. J., 40 (1991), pp. 1041-1060.

[GV93] V. A. GALAKTIONOV AND J. L. VASQUEZ, Blow-up for quasilinear heat equations described by means of nonlinear Hamilton-Jacobi equations, preprint 1993.

[HRR94a] W. HUANG, Y. REN, AND R. D. RUSSELL, Moving mesh partial differential equations (MMPDEs) based on the equidistribution principle, SIAM J. Numer. Anal., 31 (1994), pp. 709-730.

[HRR94b] _ Moving mesh methods based upon moving mesh partial differential equations, J. Comp. Phys., 113 (1994), pp. 279-290.

[HV93] M. HERRERO AND J. VELAZQUEZ, Blow-up behaviour for one-dimensional semilinear parabolic equations, Ann. Inst. H. Poincaré, Analyse nonlinéaire, 10 (1993), pp. 131-189.

[HL89] J. M. HYMAN AND B. LARROUTUROU, Dynamic rezone methods for partial differential equations in one space dimension, Appl. Numer. Math., 5 (1989), pp. 435- 450. 
[Kas77] D. KASSOY, The supercritical spatially homogeneous thermal explosion: Initiation to completion, Quart. J. Mech. Appl. Math., 30 (1977), pp. 71-89.

[LPSS86] B. LeMESURIER, G. PAPANICOLAOU, C. SUleM, AND P.-L. Sulem, The focusing singularity of the nonlinear Schrödinger equation, Phys. Rev. A, 34 (1986), pp. 1200-1210.

[Pet82] L. R. PETZOLD, A description of DASSL: A differential/algebraic system solver, SAND82-8637, Sandia Labs., Livermore, CA, 1982.

[SF90] A. M. STUART AND M. S. FlOATER, On the computation of blow-up, Euro. J. Appl. Math., 1 (1990), pp. 47-71.

[VBFZ89] J. G. Verwer, J. G. Blom, R. M. Furzeland, AND P. A. Zegeling, A moving grid method for onedimensional PDEs based on the method of lines, Adaptive Methods for Partial Differential Equations, J. E. Flaherty, P. J. Paslow, M. S. Shephard, and J. D. Vasilakis, eds., SIAM, Philadelphia, 1989. 